Biocilica

\section{ATOS SIMULTÂNEOS}

A Resolução do Conselho Federal de Medicina (CFM) n I.670/03, aprovada em I3 de junho de 2003, disciplina uma prática bastante comum entre os profissionais que trabalham com procedimentos pouco invasivos tanto diagnósticos como terapêuticos. Tomese como exemplo os exames endoscópicos realizados em clínicas e consultórios. Esses profissionais costumam assumir, simultaneamente, a responsabilidade pela sedação do paciente e a execução do exame ou do tratamento. Ao considerar como prioritária a segurança do paciente durante o procedimento e após sua realização, e a necessidade de se criar normas que definam os limites de segurança com relação ao ambiente, qualificação do pessoal, responsabilidades por equipamentos e drogas disponíveis para o tratamento de eventuais complicações, o CFM determina que os locais onde tais práticas sejam realizadas devam contar com material mínimo indispensável para o atendimento de intercorrências. Ademais, exige que "o médico que realiza o procedimento não pode encarregar-se simultaneamente da administração de sedação profunda/analgesia, devendo esta tarefa ficar a cargo de outro médico". Anexa ao corpo da citada resolução definições dos níveis de sedação e relaciona os equipamentos considerados de uso corrente nos casos de emergência e reanimação.

\section{Comentário}

Esta determinação do CFM rompe com as práticas que costumam banalizar os procedimentos mínimos na área médica. Isto é, profissionais que agem sem a devida cautela ou sensatez e assumem a responsabilidade por atos simultâneos. Segundo Gomes e França', "exceder-se na terapêutica ou nos meios propedêuticos mais arriscados é uma forma de desvio de poder e, se o dano deveu-se a isso, não há porque negar a responsabilidade profissional." Face à importância do teor da resolução urge a sua reiterada divulgação nos meios de comunicação dos conselhos. Espera-se que com a observância dessa medida, os conselhos regionais em suas ações judicantes não tenham que enfrentar o constrangimento de aplicar pena aos pares em decorrência do insucesso de atos simultâneos praticados, por violarem preceitos éticos contidos no Código de Ética Médica.

Sérgio Ibiapina F. Costa

\section{Referência}

I. Gomes JCM, França GV. Erro médico. In: Costa SI F, Garrafa V, Oselka G. Iniciação a Bioética. Brasilia: Conselho Federal de Medicina; 1998. p.243-56.

\section{Climica Cinirgica \\ CONDUTA NO CÂNCER DO RETO}

A conduta no câncer do reto continua sendo polêmica e gerando diversas opções quanto ao seu tratamento ao redor do mundo. Tentaremos dar os principais pontos de concordância para o tratamento desta doença.

Excisão total do mesorreto: deve ser realizada quando o tumor for de reto médio ou reto baixo, lembrando que esta técnica também leva a um aumento nas taxas de fístulas das anastomoses colorretais devido à desvascularização do coto retal.

Adjuvância: quimioterapia não deve ser feita em tumores TI e T2. Nos tumores T3 e $\mathrm{T} 4$ pode ser realizada, porém apresenta baixa resposta em relação a se evitar a recidiva. A radioterapia pode ser realizada quando houver invasão pélvica, no pré-operatório para diminuir a recorrência e nos idosos para realizar operações mais conservadoras.

Excisão local do câncer de reto: pode ser realizada no adenoma viloso degenerado e nos tumores TI. Nos tumores T2 e avançados não deve ser realizada, a não ser que as condições clínicas do paciente não permitirem a cirurgia e sempre com radioterapia pós operatória.

Laparoscopia no câncer de reto: pode ser feita se o tempo cirúrgico for aceitável, quando os conceitos e técnicas oncológicas forem respeitados e quando os resultados não forem comprometidos.

\section{Comentário}

Em nossa opinião, o tratamento do câncer do reto deve ser individualizado. Cada caso deve ser discutido e analisado para se optar pela melhor conduta para aquele doente em parti- cular. Como exemplo, podemos citar um indivíduo com câncer avançado de reto, passível de ser extirpado pelas condições locais pélvicas, mas sem que as condições clínicas o permitam; nesse caso pode ser uma boa indicação a químio e a radioterapia para aumentar a sobrevivência e melhorar a qualidade de vida.

\section{Elias JiRjoss Ilias \\ Osvaldo Antonio Prado Castro Paulo Kassab}

Referência

Diretrizes para o tratamento do câncer do reto. In: 52'- Congresso Brasileiro de Coloproctologia, Salvador; 2003. Resumo.

\section{Emergêneia e Medicina Yotensiva \\ HIPERTENSÃO ARTERIAL PULMONAR EM CRIANÇAS}

Até recentemente, o diagnóstico da hipertensão pulmonar primária determinava uma sentença virtual de óbito da criança, com uma média de sobrevida menor do que um ano. Nas últimas décadas houve um progresso considerável na compreensão e tratamento da hipertensão arterial pulmonar. Recentemente, Widlitz et al, 2003', realizaram uma revisão na qual colocam a compreensão atual da hipertensão arterial pulmonar com recomendações específicas relacionadas à prática terapêutica. Dentreas linhas gerais de modalidades terapêuticas, as novas drogas, por exemplo, epoprostenol, treprostinil ebosentan, eas intervenções cirúrgicas, por exemplo, septotosmia atrial e transplante têm um impacto significante na evolução e prognóstico do paciente, referindo que a Sociedade Internacional de Transplante Pulmonar e Cardíaco demonstra uma sobrevida em pediatria de $\sim 65 \%$ com dois anos póstransplante e de $\sim 40 \%$ em cinco anos (Spray et al, $200 \mathrm{I}^{2}$, nas crianças em que houve falha da terapêutica medicamentosa e da septostomia atrial. Ressaltam também' que a terapêutica vasodilatadora crônica, com bloqueadores do canal de cálcio, nos pacientes que respondem agudamente ao teste do vasodilatador $\mathrm{e} o$ uso intravenoso contínuo do epoprostenol nos que não respondem é pelo menos tão efetivo nas crianças como nos adultos, com respeito à melhora da sobrevida e dos dados hemodinâmicos, além do alívio dos sintomas. 


\section{Comentário}

As linhas gerais para o tratamento da hipertensão pulmonar ressaltam sempre a importância do diagnóstico preciso e da transferência precoce da criança para um centro de referência ${ }^{3}$. Antes do advento da terapêutica com prostaciclina, o prognóstico do paciente pediátrico era desanimador. A infusão intravenosa de prostaciclina diminui a sintomatologia e a mortalidade e, em combinação com o tratamento da hipoxemia utilizando oxigênio, é atualmente o padrão-ouro para - tratamento da criança. A prostaciclina intravenosa, além de sua ação vasodilatadora imediata, demonstrou ter um efeito remodelador e restaurador da função endotelial do vaso pulmonar. 0 tratamento com a infusão intravenosa contínua é difíil na criança e está cercada de complicações potenciais, fazendo com que o tratamento por via oral seja particularmente atrativo. Entretanto, até recentemente, esta terapêutica oral estava limitada ao uso de agentes não-seletivos, como a nifedipina, surgindo mais recentemente uma terapêutica específica (sildenafil) com efeitos secundários escassos e a possibilidade de combinação com outros fármacos 4 .

\section{Werther Brunow de Carvalho}

Referências

I. Widlitz A, Barst RJ. Pulmonary arterial hypertension in children. Eur Respir J 2003; 21:155-76.

2. Spray TL, Bridges ND. Lung transplantation for pediatric pulmonary hypertension. Prog Pediatr Cardiol 200I; 12:319-25.

3. Gibbs JSR. Recommendations on the management of pulmonary hypertension in clinical practice. Heart 200 I; 86(suppl I):il -il 3.

4. Carrol WD, Dhillon R. Sildenafil as a treatment for pulmonary hypertension. Arch Dis Child 2003; 88:827-8.

\section{Ginecologia \\ INDICAÇÕES ATUAIS DA TERAPÊUTICA HORMONAL DA MENOPAUSA}

Em maio de 2003, realizou-se na Grécia a $2^{\mathrm{a}}$ reunião do Consenso Europeu de Menopausa'; entre as conclusões, merecem destaque as indicações atuais da terapêutica hormonal da menopausa (THM): I) sintomas menopausais como ondas de calor, distúrbios do sono e secura vaginal; a dose do esteróide a ser ministrada deve ser a menor dose efetiva e prescrita exclusivamente durante a vigência dos sintomas; a via de administração deve ser aquela de preferência da mulher; 2) em mulheres de risco para osteoporose, a terapia hormonal constitui o tratamento preventivo mais simples e efetivo. Se indicada por longa duração, é fundamental que se reavalie a cada cinco anos a relação vantagens/desvantagens. É importante, também considerar outras alternativas, como os bisfosfonatos e os SERMs.

$O$ consenso reafirmou que a THM não deve ser prescrita visando à prevenção cardiovascular primária e secundária, nem a prevenção da perda de memória ou demência em mulher após a menopausa.

Nos casos de mulheres com antecedentes pessoais ou história familiar de trombose venosa antes dos 50 anos, a opinião do especialista deve ser sempre solicitada, objetivando avaliar a possibilidade de estado trombofílico. Caso indicada, a via preferencial do estrogênio deve ser a não-oral.

Nos casos de hipertrigliceridemia a via não-oral também deve ser a preferida. A relação benefício/risco deve sempre ser discutida com a paciente.

\section{Comentário}

As indicações atuais da THM após o estudo $W H{ }^{2}$ ficaram bem determinadas e este consenso é muito claro em relação a isso. As indicações são concordes com aquelas manifestadas no editorial da Ramb ${ }^{3}$, meses antes da divulgação do WHI. $O$ consenso revelou também as contra-indicações da THM que incluem porfiria cutânea, câncer de mama, tumores estrógeno-dependentes, doença cardiovascular e doença tromboembólica.

Portanto, cabe ao médico informar às suas pacientes, em diálogo franco e imparcial, os reais benefícios e riscos da THM.

José Mendes Aldrighi Ana paula S. Aldrighi marco Antonio N. Petti

Referências

I. Consensus Guidelines: menopause, State of the Art Toward Consensus. In: $2^{\text {nd }}$ European Consensus Meeting on Meno- pause;. 2003. Athens; Greece. Menopause Rev 2003; 8(2):I-7.

2. Writing Group for the Womens Health Initiative Investigators. JAMA 2002; 288:321-33

3. Editorial. Rev Assoc Med Bras 2002; 48:93-117.

\section{Obstetricia}

\section{SEGUIMENTO DE CASOS COM} DIÁSTOLE ZERO: A IMPORTÂNCIA DO PERFIL HEMODINÂMICO FETAL

A insuficiência placentária é tópico de extrema relevância no contexto obstétrico, notadamente quando os resultados da propedêutica gestacional aplicada, tal como a ocorrência de diástole zero (DZ) à dopplervelocimetria das artérias umbilicais, indicam sua intensa gravidade'. A par de incidir em gestantes com doenças graves merecedoras de cuidados específicos, acomete a gestação em tenras idades gestacionais, preceituando demandas de ações médicas altamente diferenciadas, de nível terciário. A despeito da vasta experiência observada nas publicações nacionais e internacionais, nota-se, ainda, a carência de protocolos que estabeleçam definitivamente as diretrizes para a precisa intervenção obstétrica nesses casos. Indubitavelmente, as elevadas taxas de morbidade neonatal e de mortalidade perinatal estão associadas à prematuridade extrema em conjunção com a hipoxia/anoxia fetal, as quais embaraçam a atuação obstétrica e neonatal. Se o diagnóstico dessa grave anormalidade ocorrer após a $34^{\mathrm{a}}$ semana, a interrupção imediata da gestação delineia um prognóstico neonatal muito bom ${ }^{1,2}$. Entretanto, como as complicações mórbidas neonatais aumentam com a intensidade da prematuridade, torna-se importante discutir e avaliar as medidas a serem tomadas quando o evento é verificado antes do período gestacional mencionado e, principalmente, em idades gestacionais tão precoces quanto 26 ou 27 semanas e com peso fetal estimado demasiadamente baixo, pouco acima de $500 \mathrm{~g}$. Nessas idades gestacionais, próximas da viabilidade, em face à 